\title{
Algoritmo Para El Reconocimiento Automático De La Posición De Acelerómetros En Vehículos M1
}

\section{Algorithm for Automatic Position Recognition of Accelerometers in M1 Vehicles}

\author{
Jorge Enrique Jaramillo \\ Aguilar \\ Cuenca, Ecuador \\ jjarmilloa1@est.ups.edu.ec \\ Orcid: 0000-0002-3829-1806
}

\author{
Angel Leonardo Morocho \\ Sánchez \\ Cuenca, Ecuador \\ amorochosa@est.ups.edu.ec
}

\begin{abstract}
Resumen-Este trabajo se orientó en determinar un algoritmo para reconocer de manera automática la ubicación del conector OBD II en los vehículos de categoría M1; utilizando un protocolo para la obtención de datos y como primer punto se caracterizó los espacios tridimensionales con respecto a las aceleraciones obtenidas mediante el dispositivo FREEMATICS ONE +; además, para el estudio estadístico, se obtuvo información mediante la media aritmética, habiendo seleccionado la media por su mayor aporte en la información, al facilitar la caracterización del experimento. Estos valores generaron bases de datos, mismos que se aplicaron para la manufacturación y entrenamiento de la red neuronal artificial; adicionalmente, se analizó con diferentes tipos de entrenamientos para utilizar el método de error más bajo, en el cual, mediante el entrenamiento Trainlm se alcanza un error de $0.38265 \%$. La comprobación del funcionamiento de la Red Neuronal de ubicación se realizó en 3 diferentes vehículos con los cuales se obtuvieron resultados a favor de la investigación.
\end{abstract}

Palabras Clave: OBD II, Acelerómetros, Freematics ONE+, RNA, protocolo, entrenamiento.

\begin{abstract}
This work was focused on determining an algorithm to automatically recognize the location of the OBD II connector in vehicles of category M1, using a protocol for obtaining data and, as a first point, the three-dimensional spaces were characterized, with respect to the accelerations obtained using the FREEMATICS ONE + device. In addition, for the statistical study, information was obtained, by means of the arithmetic mean, having selected the average for its greater contribution in the information, by facilitating the characterization of the experiment; these values generated databases, which were applied for the manufacturing and training of the artificial neural network. Also, it was analyzed with different types of workouts to use the lowest error method, in which through Trainlm training, an error of $0.38265 \%$ is reached. The verification of the operation of the Neural Network of location was carried out in 3 different vehicles with which there were obtained results in favor of the investigation.
\end{abstract}

Keywords: OBD II, Accelerometers, Freematics ONE +, RNA, protocol, training.

Sumario: I Introducción, II Métodos y Materiales, III Resultados y Discusión, IV Conclusiones.

Como citar: Jaramillo, Jorge., Sánchez, Angel. (2020). Algoritmo Para El Reconocimiento Automático De La Posición De Acelerómetros En Vehículos M1. Revista Tecnológica - Espol, 32(1). Recuperado a partir de http://www.rte.espol.edu.ec/index.php/tecnologica/article/view/740 


\section{INTRODUCCIÓN}

Esta investigación tuvo como objetivo determinar un algoritmo para captar la posición de los acelerómetros de manera automática en el espacio tridimensional, para lo cual, en primer momento se realizó un entrenamiento de la Red Neuronal Artificial (RNA), partiendo de una referencia inicial de los vehículos en estudio; esta posición refleja la variación de conducción con una respuesta dinámica diferente.

La dificultad de análisis y el entendimiento de los sistemas que interceden en el funcionamiento del vehículo se deben a los pocos estudios en protocolos y sistemas para obtener información precisa de los vehículos, como también el poco análisis del comportamiento de los sensores en tiempo real; por otra parte, se dificulta comprender la complejidad de las manifestaciones de los acelerómetros aplicados en el vehículo, afectando en la determinación inmediata de fallas mecánicas; asimismo, limita el mejoramiento del estudio de los ciclos de conducción. Por lo expresado, urge la utilización de nuevas técnicas para un diagnóstico eficiente.

Para enfrentar la problemática en relación al tema en estudio, se requiere determinar instrumentos que midan las aceleraciones del vehículo, que se dan por la gravedad, por la irregularidad de la calzada y por las vibraciones del motor a causa de factores internos.

Para detectar anomalías como: estado de funcionamiento incorrecto del motor y excesivas emisiones contaminantes, resulta esencial la utilización del acelerómetro para proporcionar resultados en beneficio de la tecnología automotriz de manera continua, a través de los métodos de diagnóstico, admitiendo un mantenimiento apropiado que conlleva a disminuir costos y contaminación ambiental generada [1].

La investigación de Dávila [2] muestra el boceto de un modelo electrónico con el uso de un acelerómetro para mejorar el control de velocidad y estabilidad dinámica; en igual forma Händel y Enstedt [3] proponen un diseño para combatir el efecto sentadilla del chasis basado en un modelo paramétrico que describe el efecto de la inclinación del vehículo en las mediciones del acelerómetro en función de la aceleración horizontal.

Con el acelerómetro alineado verticalmente se puede determinar la odometría de un vehículo; al respecto, Gutiérrez et al. [4] presentan una nueva aplicación con este instrumento que, en lugar de usarlos para medir la aceleración lineal del vehículo, lo aplican para medir cuatro posiciones de giro de la rueda, cuya función es determinar la orientación gradual del neumático en movimiento; mientras que la presente investigación se ocupó de utilizar el acelerómetro para determinar la posición del conector OBD II.

Mediante un acelerómetro se puede obtener la posición precisa de un vehículo; al respecto, Eger y Kiencke [5] hacen referencia de los giroscopios para medir la velocidad angular, mismos que pueden ser reemplazados por señales de aceleración que de paso abarata costos. Otra importante aplicación de los acelerómetros es la estimación de la velocidad angular como lo muestran Lee et al. [6], quienes realizan la estimación de la velocidad angular a partir de las salidas de un acelerómetro. Joubert y Beer [7] en su investigación utilizan los datos del acelerómetro y velocidad del vehículo para rastrear el comportamiento de conducción aceptable y no aceptable y calcular un modelo de riesgo más completo, utilizando los datos y no umbrales de comportamiento de conducción; este aporte sirvió para comprender los ciclos de conducción, cuyo conocimiento fue transferido para la obtención de datos eficientes en esta investigación.

Ayad et al. [8] señalan que la detección de fenómenos en relación con la combustión, como: fallas de encendido o golpes de pre-ignición es necesaria para el desarrollo de controles electrónicos. Sin embargo, a través de acelerómetros se pueden realizar distintas aplicaciones para detectar el impacto de la combustión en la vibración de la estructura del motor.

En la actualidad, los giroscopios se están implementando en sistemas inteligentes del vehículo para mejorar su rendimiento, Zhu et al. [9], plantean un método de identificación de la presión de los neumáticos inteligentes, utilizándolos con un acelerómetro de tres ejes; de igual manera Vladimir et al. [10], desarrollan un complejo hardware y software que incorpora un acelerómetro para el registro y análisis de los parámetros de movimiento de un vehículo.

Los acelerómetros también se utilizan como dispositivos de seguridad, como es el caso de los sensores de choque que son los elementos encargados de detectar el impacto y generar una señal que se envía a la unidad de control electrónico del sistema para activar el airbag y/o el dispositivo pirotécnico de los cinturones de seguridad [11][12].

\section{MÉTODOS Y MATERIALES}

A continuación, se desenvuelven los argumentos primordiales referentes a la elección del equipo práctico e instrumentación mínimamente invasiva, escenarios de obtención de datos, sistemática de datos, manufactura de matriz de propiedades, disminución de propiedades y creación de la Red tipo Feed-forward backprop.

\section{A. Elección del equipo experimental e instrumentación minimamente invasiva}

Para la elección del equipo experimental en referencia al Freematics ONE+ que conforma el sistema instrumental, se procedió a establecer un protocolo que consistió en lo siguiente: identificar una superficie plana para encontrar el punto de inicio con $0^{\circ}$ en los 3 ejes $(\mathrm{X}, \mathrm{Y}, \mathrm{Z})$ del espacio tridimensional y como punto de partida se tiene el vehículo, mismo que en estado estático o en movimiento emite información a través de la extensión OBD II hacia el dispositivo Freematics ONE+; y este a su vez, se apoya del giroscopio para variar los datos de aceleración, los cuales fueron almacenados y enviados al ordenador para proceder al aprendizaje de RNA. Por lo expuesto, se explica que la elección de los elementos del sistema se dio en base al análisis del funcionamiento del acelerómetro aplicado en otras investigaciones, como es el caso del aporte de Vladimir et al. [10], quien en su investigación incluyo un 
acelerómetro para el registro y análisis de los parámetros de movimiento de un vehículo.

Luego de compactar el sistema de acoplamiento, mediante el protocolo referido en párrafo anterior para la creación de la RNA, se realizaron aplicaciones de prueba y error, hasta obtener los datos adecuados para la proyección del algoritmo. Cabe señalar que es imprescindible desconectar la extensión OBD II del vehículo para conseguir los datos de los acelerómetros.

Explicando el proceso interno, en cuanto al funcionamiento del Freematics $\mathrm{ONE}+$, se detalla lo siguiente:

Cada ángulo se determinó por medio de un goniómetro, mientras que el reconocimiento de las aceleraciones se consiguió mediante el dispositivo Freematics One + , mismo que se enlazó al puerto OBD II de un automóvil y trabajó como un módulo inteligente e independiente; además, el instrumento en referencia tiene agregado un sensor de movimiento (acelerómetro, giroscopio) permitiendo generar varias aceleraciones que se procesan y se registran en tiempo real, guardándose en una microSD interna y enviarse por medio de WiFi o red. Este proceso tuvo como referencia el manual de Freematics [13].

En la Fig. 1 se presenta: el equipo experimental que se puso a prueba en un vehículo Chevrolet Aveo 1.6, la extensión requerida para OBD II, la unidad Freematics One + , un estabilizador de cámara (giroscopio), una tarjeta de memoria microSD y la computadora (PC Laptop). Estos elementos aportan en obtener información válida de la matriz de atributos para la RNA.

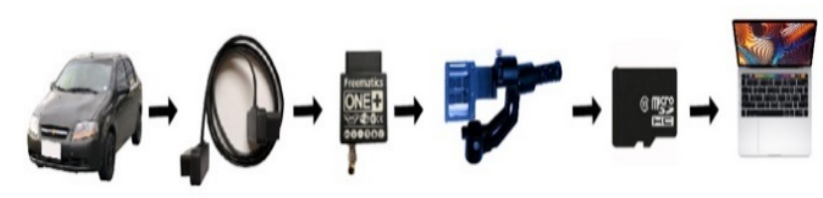

Fig. 1 Elementos de muestreo

\section{B. Escenarios de obtención de datos}

Se utilizó el software Matlab junto al dispositivo Freematics One+ para la producción de datos. Para dicha tarea de recolección de datos se requiere que el motor se encuentre en ralentí, alrededor de $850 \mathrm{rpm}$.

Realizando un pre-experimento se determinó que los datos de las aceleraciones conseguidas poseen picos o cimas de mayor frecuencia, debido a las excitaciones generadas por el motor del automóvil, optando por la opción de realizar la toma de datos de manera externa al vehículo sobre una zona plana. Los datos se obtienen mediante el terminal OBD II y se procesan a una frecuencia de 50 a $60 \mathrm{~Hz}$ durante 5 minutos, con una diversificación de $2.5^{\circ}$ grados, iniciando desde $0^{\circ}$ hasta $180^{\circ}$ grados hacia los ejes X, Y; adicional a esto y conjuntamente, se ejecutó la toma de muestras formando una combinación de los tres ángulos.

\section{Sistemática de datos}

En la Fig. 2 se exponen los dispositivos físicos manipulados para la obtención y acaparamiento de datos.

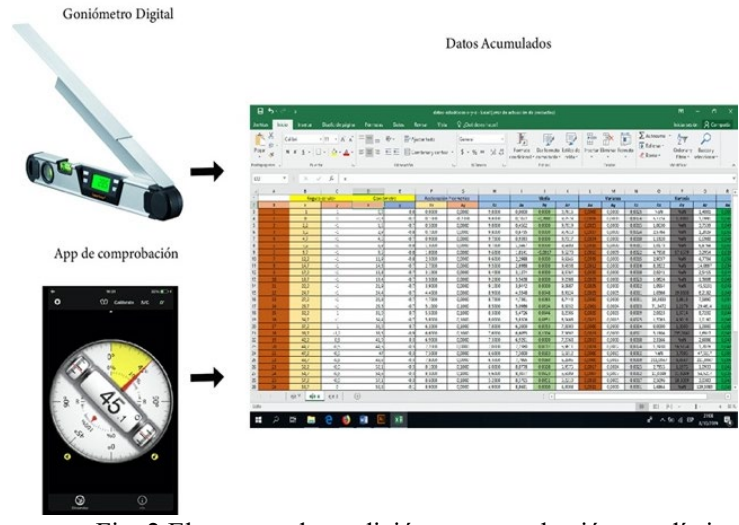

Fig. 2 Elementos de medición y comprobación estadística

Para adquirir las aceleraciones con el módulo Freematics One + , se continúa el flujograma de proceso que se exhibe en la Fig. 3.

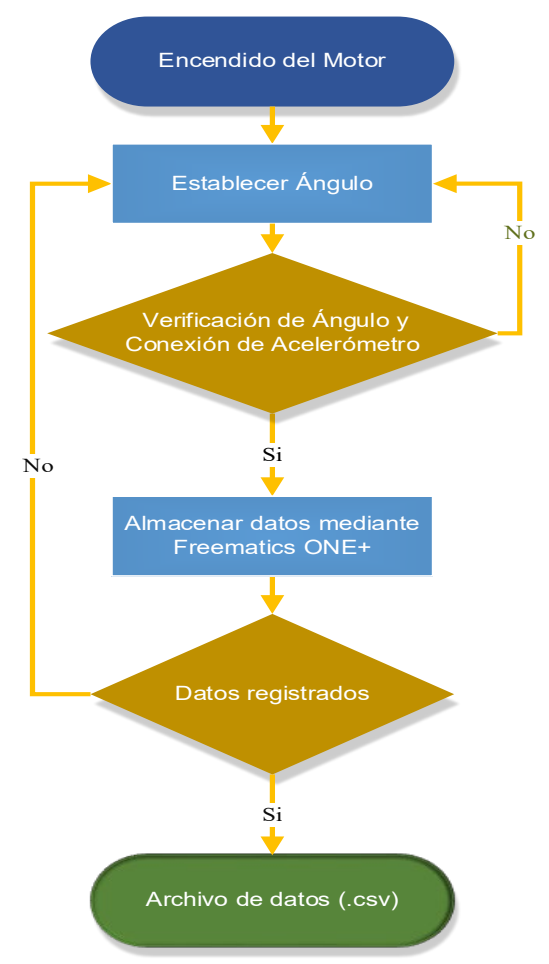

Fig. 3 Flujograma de muestreo

La adquisición de datos se prepara encendiendo el motor del vehículo; posterior a esto, se constituyen los ángulos para su verificación y conexión de acelerómetro; al cumplirse estas condiciones se almacenan los datos con el dispositivo Freematics One+ en la tarjeta microSD, la cual guarda dicha adquisición en un archivo Excel, el mismo que emite un archivo donde las variables son separadas mediante comas (.CSV). De no resultar la operación, se procede a la verificación de la conexión del terminal

\section{Manufactura de matriz de propiedades}

Habiendo logrado la adquisición de datos, se procede a efectuar la lectura de éstos en el archivo Excel; la Tabla 1 muestra una fracción de los datos que se obtienen a partir del dispositivo Freematics One+. Para el análisis de la presente investigación, se toma como referencia las aceleraciones, las cuales están determinadas por un número 
veinte (20) que inicia espaciado por una coma (,), el cual da la aceleración en $\mathrm{X}\left(26 \frac{\mathrm{m}}{s^{2}}\right), \mathrm{Y}\left(56 \frac{\mathrm{m}}{s^{2}}\right), \mathrm{Z}\left(-76 \frac{\mathrm{m}}{s^{2}}\right)$, mismas que se diversifican dependiendo del movimiento y posición espacial; es decir, obedeciendo la ubicación del acelerómetro. Ver TABLA I.

TABLA I

APARCAMIENTO DE DATOS
\begin{tabular}{|r|}
\hline \multicolumn{1}{|c|}{0,10246} \\
\hline $10 \mathrm{D}, 0$ \\
\hline $10 \mathrm{C}, 851$ \\
\hline $10 \mathrm{~F}, 40$ \\
\hline $10 \mathrm{~B}, 29$ \\
\hline 105,95 \\
\hline 106 \\
\hline 107,24 \\
\hline 114,104 \\
\hline $20,26,56,-76$ \\
\hline
\end{tabular}

\section{E. Disminución de propiedades}

En la Fig. 4 se demuestra que los datos, expresados a través de los acelerómetros del dispositivo Freematics One+, tienen diversas alteraciones en las señales, provocadas por las vibraciones externas o ambientales; por lo que, los datos que se emplearon para el adiestramiento de la RNA, fueron obtenidos mediante el diseño del filtro Savitzky-Golay a partir de un aproximado de 250 datos, que permitió disminuir los picos altos y bajos en los ejes de $\mathrm{X}, \mathrm{Y}$ y Z de la siguiente manera:

- Se utilizó el algoritmo planteado por Gárate [14]

- En base al algoritmo se segregaron los datos de aceleración para aplicar el filtro Savitzky-Golay.

- Por otra parte, para obtener los datos de la media Aritmética, se tomó como referencia los resultados del filtro Savitzky-Golay para obtener un solo dato valido por cada eje tridimensional, que fue reducido a través del algoritmo planeado por Gárate para la red de aprendizaje.
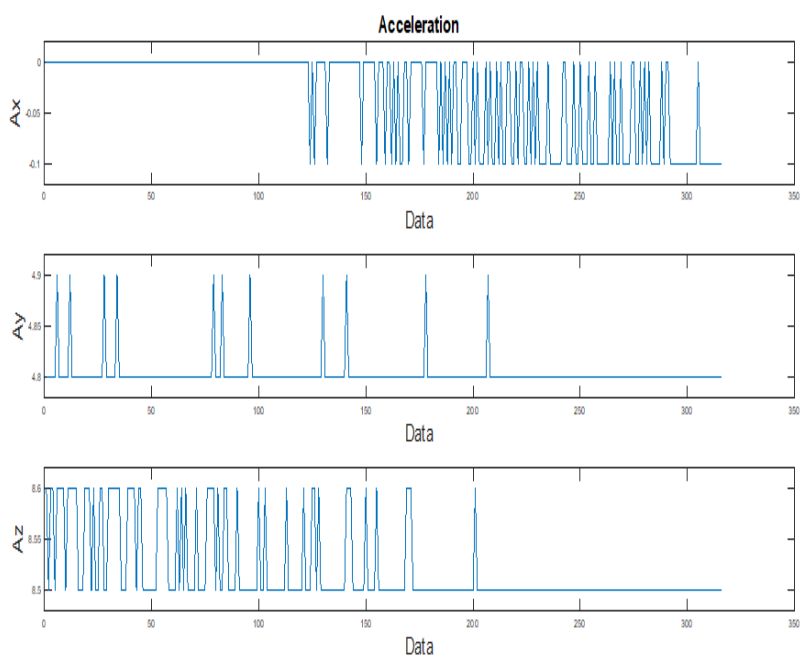

Fig. 4 Datos de aceleraciones Ax,Ay,Az.

\section{F. Creación de la Red tipo Feed-forward backprop}

En la elección de la red neuronal de tipo Feed-forward backprop, se establecieron aplicaciones prácticas con diferentes tipos de redes neuronales, en la cual se manejaron desiguales cuantificaciones de adiestramiento como:

- Tipo de red.

- Función de entrenamiento.

- Función de aprendizaje de adaptación.

- Rendimiento de la función.

- Número de capas.

- Cantidad de neuronas.

- Función de transferencia.

En la Fig. 5 se presentan las fallas presentadas durante los adiestramientos aplicados con distintas clases de RNA, en donde se escoge la red que posea un menor error durante el diseño del algoritmo.

\section{ERRORES DE ENTRENAMIENTO}

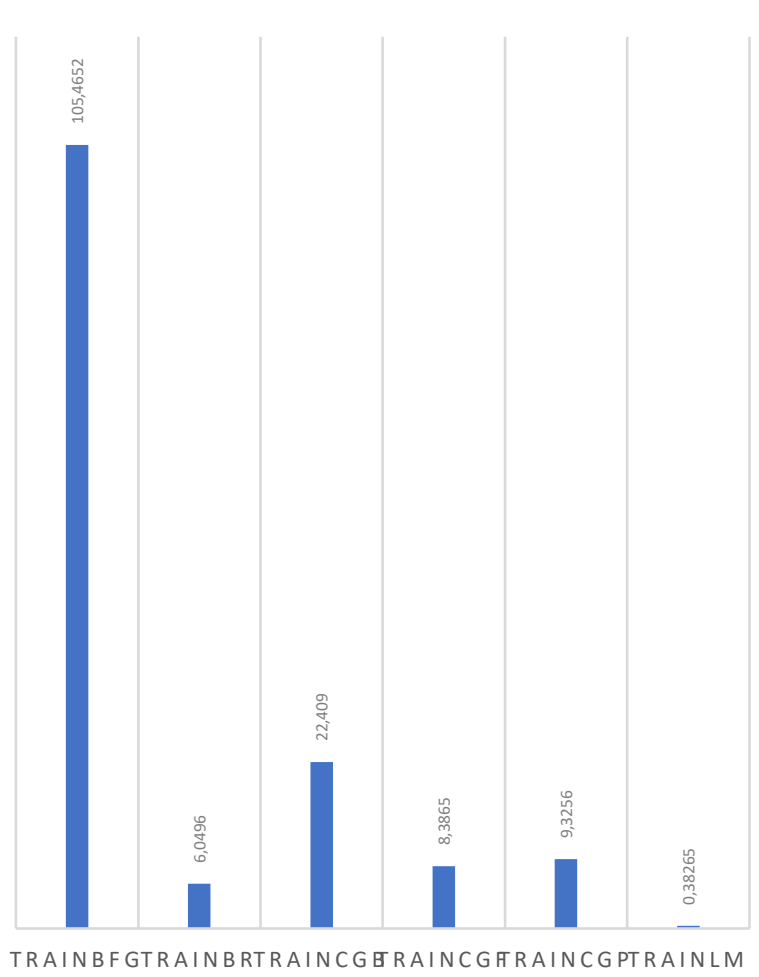

Fig. 4 Errores con varias funciones de entrenamiento

En la Fig. 6 se muestran las características de la RNA tipo 'Feed-forward backprop' adiestrada con una función de preparación Levenberg-Marquardt (trainlm), en el cual se enseña el suministro de la red con tres medidas de ingreso, atravesando dos capas ocultas y una capa de salida, en donde las dos primeras están establecidas con cuarenta neuronas además de una función de transferencia 'Tansig', mientras que en la capa final se utiliza una función de transferencia tipo 'Purelin'; en último lugar la red envía una señal de salida de datos con los ángulos referentes a los tres ejes de aceleraciones. 


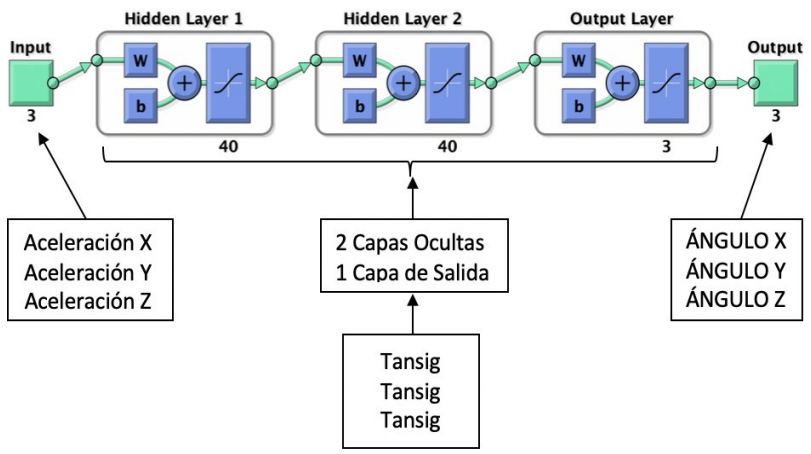

Fig. 5 Estructura de red neuronal

En la Fig. 7 se presenta el gradiente en la disminución del error medio cuadrático o MSE y además se representa el número de épocas aplicadas.

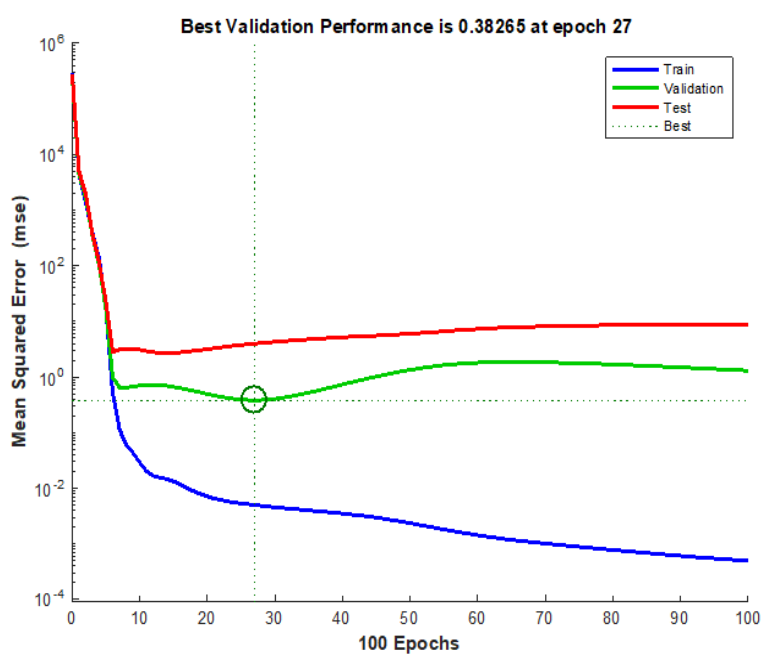

Fig. 6 Rendimiento de validación

Adicional a esto, en la Fig. 8 se indica el progreso del adiestramiento con una ratificación de 73 puntos dentro de la RNA.
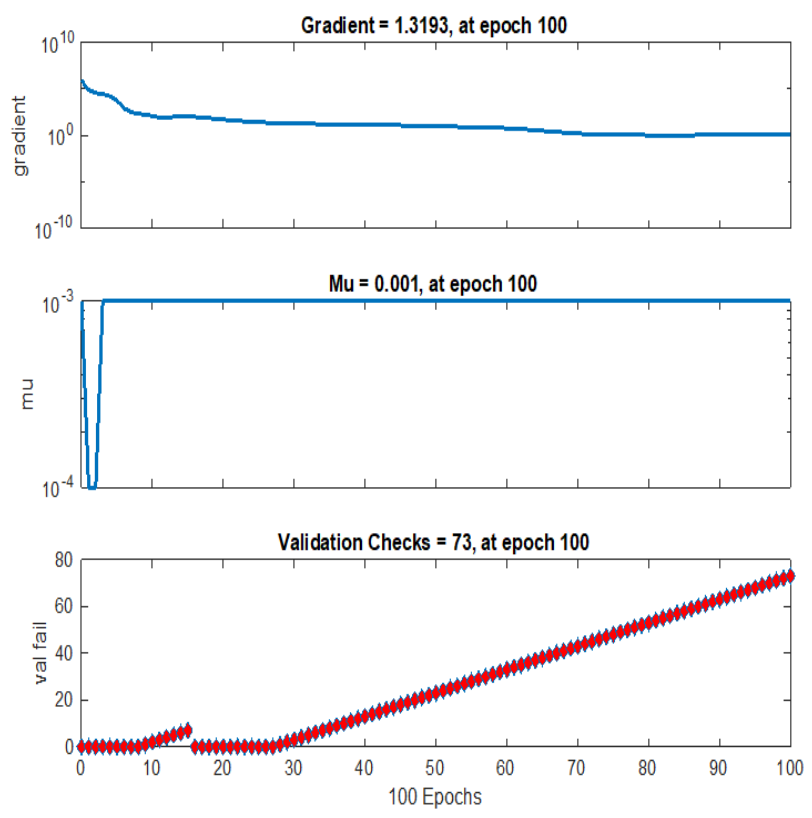

Fig. 7 Rendimiento de validación
En la Fig. 9 se indican los siguientes resultados obtenidos del factor de correspondencia de Pearson R de la red neuronal manufacturada. Las líneas indican los valores esperados, en cambio los círculos negros representan los valores obtenidos por la RNA. La predicción obtenida por la red neuronal es eficaz y se evidencia con un buen desempeño, dado que se consigue un índice global cercano a uno en: entrenamiento, validación y prueba.
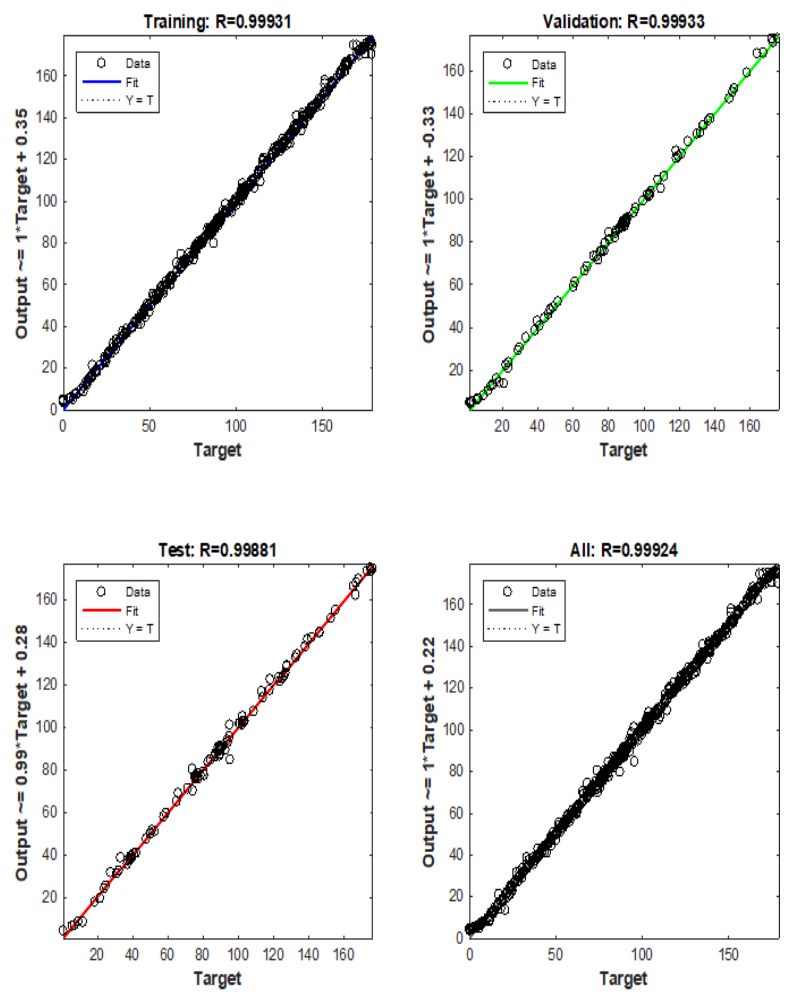

Fig. 8 Correlación entre los valores esperados y pronosticados por la red neuronal

\section{RESULTADOS Y DISCUSIÓN}

Con la finalidad de analizar el correcto funcionamiento de la RNA manufacturada y entrenada, se realizan 3 pruebas en vehículos de categoría M1. En la TABLA II se detalla la ubicación de los acelerómetros de los vehículos en prueba que fueron en un Hyundai Tucson IX, año 2014 y Volkswagen Gol, año 2011, y además el vehículo Chevrolet Aveo, año 2009, que se utilizó para realizar la investigación de los ángulos respectivos.

TABLA II

FRACCIÓN DE LA MATRIZ COMPARATIVA DE RESULTADOS

\begin{tabular}{|c|c|c|c|c|c|c|}
\hline \multirow[t]{2}{*}{$\begin{array}{l}\text { Vehículos } \\
\text { categoría } \\
\text { M1 }\end{array}$} & \multicolumn{3}{|c|}{$\begin{array}{l}\text { Ángulos emitidos } \\
\text { por la red } \\
\text { neuronal (grados) }\end{array}$} & \multicolumn{3}{|c|}{$\begin{array}{l}\text { Ángulos tomados } \\
\text { de manera } \\
\text { manual. (grados) }\end{array}$} \\
\hline & $A x$ & Ay & $\mathrm{Az}$ & Ax & Ay & $\mathrm{Az}$ \\
\hline $\begin{array}{l}\text { Hyundai } \\
\text { Tucson IX } \\
2014\end{array}$ & 86.4 & 69.8 & 25.4 & 84.7 & 72.6 & 18.2 \\
\hline $\begin{array}{l}\text { Volkswagen } \\
\text { Gol 2011 }\end{array}$ & 86.7 & 49.3 & 45.4 & 87.9 & 44.9 & 45.1 \\
\hline $\begin{array}{l}\text { Chevrolet } \\
\text { Aveo } 2009\end{array}$ & 92.1 & 89.3 & 175.4 & 93.6 & 89.4 & 176.3 \\
\hline
\end{tabular}


Con el resultado obtenido de la investigación, se estima que la confianza es del $99.61735 \%$ puesto que, en el entrenamiento Trainlm se obtiene un error del $0.38265 \%$.

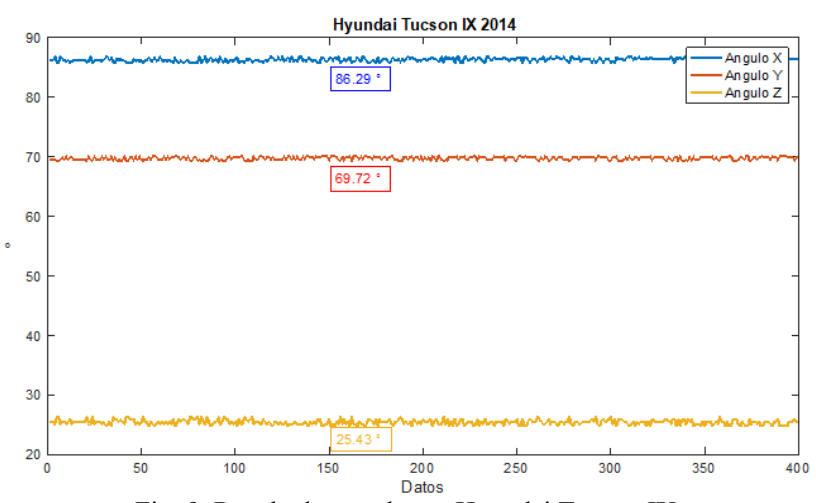

Fig. 9 Resultado angular en Hyundai Tucson IX

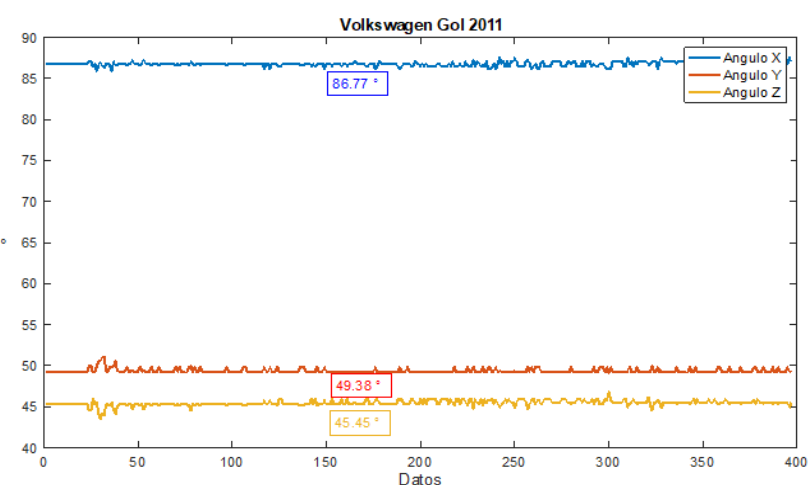

Fig. 10 Resultado angular en Volkswagen Gol

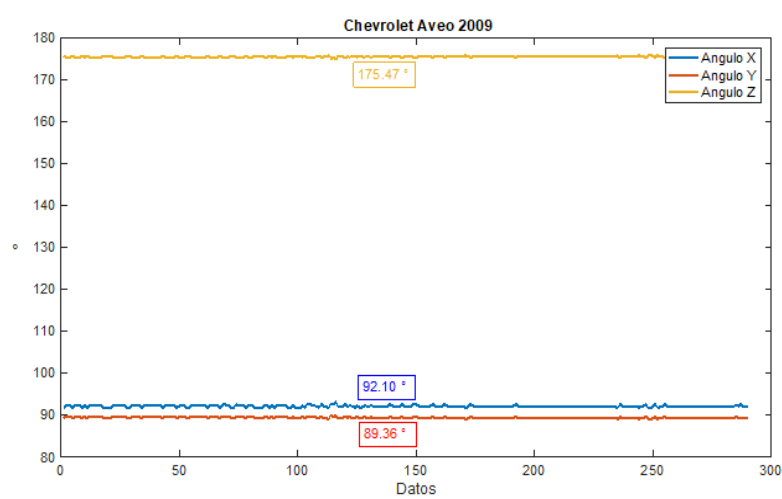

Fig. 11 Resultado angular en Chevrolet Aveo

En las Fig. 10, Fig. 11 y Fig. 12 que anteceden, se muestran las ubicaciones espaciales de los acelerómetros de tres vehículos categoría M1, que fueron utilizados para detectar el reconocimiento de la posición del puerto OBDII; y para el efecto se creó un algoritmo que proporcionó resultados de manera automática; esto es la posición en grados $\left(^{\circ}\right)$.

Continuando con el análisis de las tres figuras, resulta positivo comparar las tres distintas dimensiones de los ejes tridimensionales, evidenciando las diferencias en las ubicaciones de los puertos OBDII que existen en los 3 vehículos, según su modelo. Este diagnóstico representa el logro planteado de la presente investigación.

Como nota adicional, durante el proceso de transformación se encontraron problemas por motivo de las vibraciones y la determinación del plano exacto donde se encontraba el vector de aceleración.

\section{CONCLUSIONES}

El método estadístico que se utilizó para obtener la información necesaria que dirigió al aprendizaje de la RNA fue en primer momento un aparcamiento de datos mediante uso de filtro Savitzky-Golay, dando paso al segundo método aplicado, que consistió en la media aritmética por su menor porcentaje de falla.

Se comprobó la veracidad de los ángulos en 3 dimensiones mediante el uso de cosenos directores en conjunto a las leyes trigonométricas de los triángulos.

Se obtuvo la corrección adecuada de la RNA al momento en que se aplicó un filtro en la matriz de aprendizaje, puesto que, como previo se menciona las calzadas de irregularidades que producen valores no previstos o no lógicos para el adiestramiento de la ya mencionada red neuronal; esto a su vez, garantiza un correcto estado para la toma de datos y así obtener resultados mucho más fiables.

Se basó en el valor positivo y negativo de los datos para la organización de los mismos en la RNA, utilizando todas las combinaciones posibles en lo que respecta $\mathrm{a}+\mathrm{y}-$.

A través de los acelerómetros se obtuvo la ubicación exacta del DLC en las 3 dimensiones utilizables, mediante el uso de aceleraciones convertidas a grados, utilizando el algoritmo.

El algoritmo determinado para captar la posición de los acelerómetros de manera automática en el espacio tridimensional resultó de una codificación para llegar a establecer los ángulos de posición de los vehículos con OBD II, para lo cual se analizaron todas las posiciones que se generaron durante los ensayos prueba-error con el dispositivo Freematics ONE+.

La presente investigación también facilita en un futuro abrir paso a nuevas investigaciones en las que se pueda involucrar el estado del motor, estado de la suspensión e incluso de la calzada, únicamente mediante el uso del análisis de vibraciones; como también, la información obtenida servirá en lo posterior para mejorar los diagnósticos, reduciendo el tiempo y costos de mantenimiento.

\section{REFERENCIAS}

[1] Kyrtsos, C. T. (24 de 09 de 1998). UNITED STATES Patente $n^{\circ}$ US 6,225,894 B1.

[2] Miranda Davila, R. J. (2019). Diseño de un prototipo electrónico utilizando sensores acelerómetro y giroscopio para optimizar el control de velocidad y estabilidad dinámica de un vehículo. Lima: Universidad Técnologica de Peru.

[3] Peter, H., \& Martin, B. E. (2019). Combating the effect of chassis squat in vehicle performance calculations by accelerometer measurements. ScienceDirect, 43(Issue 4), 483-488.

[4] Joaquin Gutírrez, M. A., \& Porta Gandara, F. (2010). Vertically aligned accelerometer for wheeled vehicle odometry. ScienceDirect, 20(5), 617-625.

[5] Ralf, E., \& Maisch, A. (2000). Estimation of Rotational States in Vehicle Rollover Using Accelerometers. ScienceDirect, 33(11), 553-558.

[6] Lee, J., Kim, H., Oh, S., Do, J., Nam, C., \& Hwang, D. (2019). Lee, J. a , Kim, H. b, Oh, SH c , Do, JC d, Nam, CW d, Hwang, D.-H. segundoAutor de correo electrónico , Lee, SJ b. MicroSystem Thecnologies, 25(7), 2855-2867.

[7] Joubert, J., Koker, N., \& Dirk, B. (2016). Combining accelerometer data and contextual variables to evaluate the risk of driver behaviour. ScienceDirect, 41(A), 80-90. 
[8] Ayad, S., Sharma, S., Verma, R., \& Henein, N. (2019). Combustion Ionization for Detection of Misfire, Knock, and Sporadic Preignition in a Gasoline Direct Injection Engine. Journal of Energy Resources Technology, Transactions of the ASME, 141(11).

[9] Zhu, B., Han, J., \& Zhao, J. (2019). Tire-pressure identification using intelligent tire with three-axis accelerometer. Sensors, 19(11).

[10] Vladimir, B., Iurii, N., Artem, M., Stanislav, S., \& Egor, G. (2019) Development of a hardware-software complex for automatic registration and analysis of vehicle motion parameters. Scopus, 2136-2138.

[11] Naylam Mechatronic. (s.f.). Recuperado el 25 de 08 de 2019, de https://naylampmechatronics.com/blog/45_Tutorial-MPU6050Acelerómetro-y-Giroscopio.html

[12] Pablo Luque, D. A. (2004). Ingenieria del Automovil sistemas y comportamiento dinamico. Madird.

[13] Freematics. (s.f.). Freematics. Recuperado el 10 de 06 de 2019, de https://freematics.com

[14] Gárate Montalvo, D. A. (Noviembre de 2018). Desarrollo de un algoritmo para la adquisición y almacenamiento de datos provenientes del sistema de diagnóstico a bordo OBD II. Cuenca, Azuay, Ecuador. 